\title{
Do We Understand Magnetic Properties of $\mathrm{ZnMnO}$ ?
}

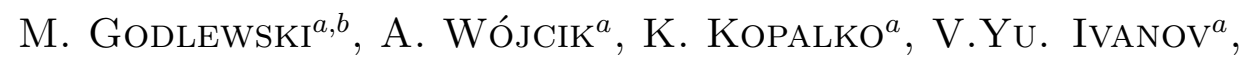
Z. Wilamowski ${ }^{a}$, R. Jakięa ${ }^{a}$, E. GuZIEWICZ $^{a}$, A. Szczepanik ${ }^{a}$, P. DŁużewski ${ }^{a}$, E. Chikoidze ${ }^{c}$, J. Barjon $^{c}$, Y. Dumont ${ }^{c}$, M. Putkonen ${ }^{d, *}$, L. Ninnistö ${ }^{d}$ And Dong TAng ${ }^{e}$

${ }^{a}$ Institute of Physics, Polish Academy of Sciences al. Lotników 32/46, 02-668 Warsaw, Poland

${ }^{b}$ Dept. Mathematics and Natural Sciences, College of Science Cardinal S. Wyszyński University, Warsaw, Poland

${ }^{c}$ Groupe d'Etudes de la Matière Condensée, CNRS-Université de Versailles 45 avenue des Etats-Unis, 78035 Versailles, France

${ }^{d}$ Laboratory of Inorganic and Analytical Chemistry Helsinki University of Technology P.O. Box 6100, 02015 Espoo, Finland

${ }^{e}$ FEI Co, 5600 MD Eindhoven, Netherlands

\begin{abstract}
Optical and magnetic properties of $\mathrm{ZnMnO}$ films are discussed based on the results of cathodoluminescence, photoluminescence, and magneto-photoluminescence investigations. We show that photoluminescence/cathodoluminescence emissions are strongly quenched and become in-plane inhomogeneous in samples with increased Mn fractions. Strong polarization of photoluminescence is observed, even though excitonic lines do not shift and are not split at magnetic fields up to $6 \mathrm{~T}$.
\end{abstract}

PACS numbers: 81.15.Gh, 75.75.+a, 78.30.Fs, 78.60.Hk

\section{Introduction}

Room temperature (RT) ferromagnetism (FM) was predicted for $p$-type $\mathrm{ZnMnO}$ [1]. However, $\mathrm{FM}$ of $\mathrm{ZnMnO}$ (and also of $\mathrm{ZnCoO}$ ) reported till now

*Present address: Beneq Oy, Ensimmäinen savu, 01510 Vantaa, Finland. 
was due to inclusions of various $\mathrm{Mn}(\mathrm{Co})$ oxides and to metal accumulations and not to volume properties of $\mathrm{ZnMnO}(\mathrm{ZnCoO})$ alloys [2-5]. Such inclusions and accumulations are present in most of $\mathrm{ZnMnO}$ films grown with high temperature methods, giving a dominant magnetic response of these $\mathrm{ZnMnO}$ samples.

Our recent investigations indicate that growth at low temperature (LT) is the most promising way to avoid the formation of foreign phase inclusions of $\mathrm{Mn}_{x} \mathrm{O}_{y}$ in $\mathrm{ZnMnO}$ and to block a spinodal decomposition [3-8]. LT ZnMnO films were grown by either atomic layer deposition (ALD) [3-5] or metalorganic vapor phase epitaxy (MOVPE) [6-8]. These LT ZnMnO films, when grown with low Mn fractions, are practically inclusions free, show homogeneous Mn distribution, and are paramagnetic [5].

In this presentation we discuss optical, magnetic and magneto-optical properties of LT ZnMnO films grown by ALD and MOVPE. Results of cathodoluminescence (CL), photoluminescence (PL), magneto-PL investigations are discussed. We show that PL and CL of $\mathrm{ZnMnO}$ are observed only for samples with low $\mathrm{Mn}$ fractions and that intensity of PL/CL emissions drops rapidly with an increasing $\mathrm{Mn}$ content, indicating that $\mathrm{Mn}$ acts as emission deactivator in $\mathrm{ZnMnO}$. In the samples with an increased Mn content PL/CL becomes in-plane inhomogeneous. LT ZnMnO films grown with low Mn fractions (below 5\%) are paramagnetic and show at LT a weak antiferromagnetic contribution due to close Mn-Mn pairs.

\section{Samples}

The present investigations were performed on $\mathrm{ZnMnO}$ epilayers grown by ALD or MOVPE at LT (below $500^{\circ} \mathrm{C}$ ) using organic zinc and manganese precursors.

In ALD we employed the following metalorganic zinc and manganese precursors: zinc acetate $\left(\mathrm{Zn}\left(\mathrm{CH}_{3} \mathrm{COO}\right)_{2}\right)$ or diethylzinc (denoted as DEZn) for zinc, and two types of Mn precursors: manganese-tris-2,2,6,6-tetramethyl-3,5- heptanedione (denoted as $\left.\mathrm{Mn}(\text { thd })_{3}\right)$ and tris(2,4-pentanedionato) manganese(III) (denoted as $\left.\mathrm{Mn}(\mathrm{acac})_{3}\right)$. As an oxygen precursor we used water vapor or ozone. Temperatures of precursors were as follows: $230-250^{\circ} \mathrm{C}$ for zinc acetate, room temperature for $\mathrm{DEZn}, 160^{\circ} \mathrm{C}$ for $\mathrm{Mn}(\mathrm{acac})_{3}$, and $160-180^{\circ} \mathrm{C}$ for $\mathrm{Mn}(\text { thd })_{3}$. Substrate ((0001) sapphire or (0001) sapphire/GaN) temperature was selected in the range of $160-360^{\circ} \mathrm{C}$. Pressure in a growth chamber, i.e., a pressure of $\mathrm{N}_{2}$ transport and purging gas, was a few mbar. Ratio of Zn-to-Mn ALD cycles was either 9 to 1 or 10 to 1 , which we found to be optimal for a growth of fairly depth homogeneous $\mathrm{ZnMnO}$ films (with $0.6-0.8 \mu \mathrm{m}$ thickness) with low Mn fractions [3, 5].

MOVPE samples (3 $\mu \mathrm{m}$ thick) were grown on (0001) sapphire or silica using DEZn and tertiary-butanol as zinc and oxygen precursors. Liquid tri-carbonyl-methylcyclopentadienyl-manganese $\left((\mathrm{CO})_{3} \mathrm{CH}_{3} \mathrm{C}_{5} \mathrm{H}_{4} \mathrm{Mn}\right)$ was used as manganese precursor and hydrogen as a vector gas. The substrate temperature was $450^{\circ} \mathrm{C}$, which was optimal for obtaining high crystallographic quality samples [6-8]. 


\section{Optical and magneto-optical characterization}

The CL spectra were taken either at RT or at liquid nitrogen temperature with Jeol JSM-840 scanning electron microscope (SEM). A HORIBA JOBIN YVON SAS system was used to collect the CL emission. The light was collected by a parabolic mirror and injected via an achromatic optical bench into a TRIAX550 monochromator equipped with an UV enhanced-silicon CCD camera.

PL and magneto-PL investigations were performed at $2 \mathrm{~K}$ with samples mounted in a Spectromag 6000 split coil superconductive magnet system of Oxford Instruments. He-Cd laser PLASMA model HCCL-15UM was used for PL excitation and PL spectra were detected with a double monochromator LOMO MDR-23 equipped with Hamamatsu S7035 CCD camera and Hamamatsu photon counting system with R2531 PMT and FAST ComTec 7887 card.

\section{Results and discussion}

We have reported the first puzzling results of PL and magneto-PL investigations of $\mathrm{ZnMnO}$ (for bulk sample) in Ref. [9]. We reported that visible PL was not observed for most of the studied by us bulk $\mathrm{ZnMnO}$ samples. We could detect visible PL only in samples containing low Mn fraction (typically about 5\% and less) [9]. For such samples no spectral shifts and well-resolved splitting were observed for magnetic field up to $7 \mathrm{~T}$. Such shifts (by few $\mathrm{meV}$ ) and splitting were expected due to strong magneto-optical effects commonly seen in diluted magnetic semiconductor (DMS) samples.

We obtained similar PL results for $\mathrm{ZnMnO}$ layers studied in the present work. At low temperature PL (Fig. 1) and CL spectra were dominated by bound excitonic emissions. "Band edge" part of $2 \mathrm{~K}$ PL is shown in Fig. 1 for three MOVPE films with nominal Mn fractions of $0.6,2$, and $7 \%$. One can see that the $\mathrm{PL}$ intensity is relatively low, as compared to the intensity of PL emission of the reference bulk sample, and, moreover, drops rapidly with an increasing Mn fraction in ZnMnO films. PL was observed up to RT for MOVPE and ALD samples grown with low Mn fractions. The ratio of band edge to red emissions depended on the sample and on temperature. Red PL becomes relatively intense (as compared to the excitonic emission) at increased temperatures.

We observed similar spectra and their dependences in CL investigations. We observed that for samples with a larger Mn content CL becomes in-plane inhomogeneous, as we show in Fig. 2 for MOVPE $\mathrm{ZnMnO}$ film with a relatively large Mn fraction (18.8\%). There, the CL emission comes only from bright spots (see Fig. 2b). A comparison of SEM and CL images indicates that these bright spots come mostly from the regions of dislocations, suggesting their decoration with impurities. Otherwise emission is either very weak or is not observed. This leads us to the conclusion that Mn acts as a "killer" of a visible PL in ZnMnO, as was also proposed in Ref. [10]. 


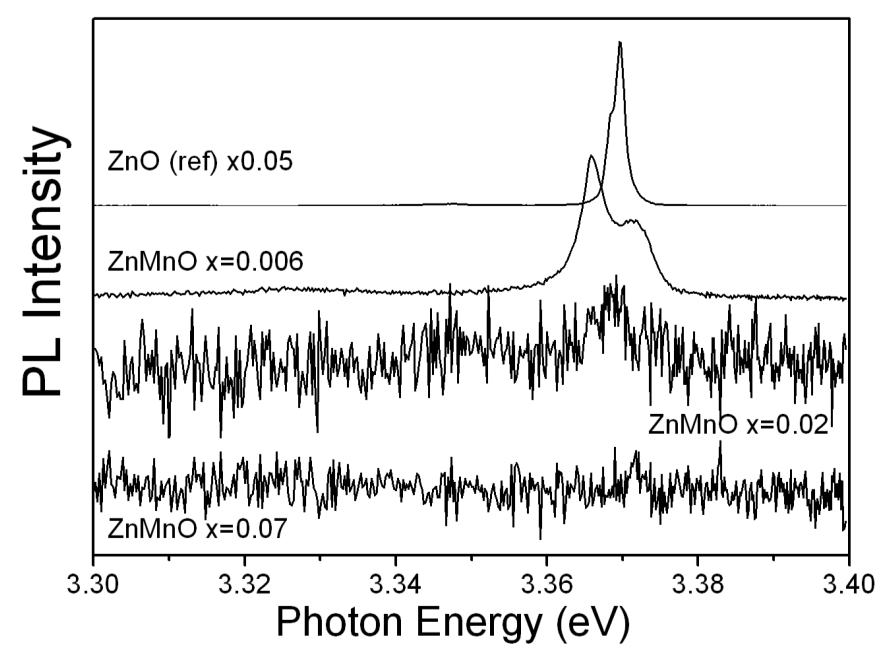

Fig. 1. Low temperature photoluminescence of $\mathrm{Zn}_{1-x} \mathrm{Mn}_{x} \mathrm{O}$ MOVPE films with three different Mn fractions $(x=0.006,0.02$, and 0.07), as indicated in the figure. PL of the reference bulk $\mathrm{ZnO}$ sample is shown for the comparison. PL intensity for this sample was scaled down by factor 20 .
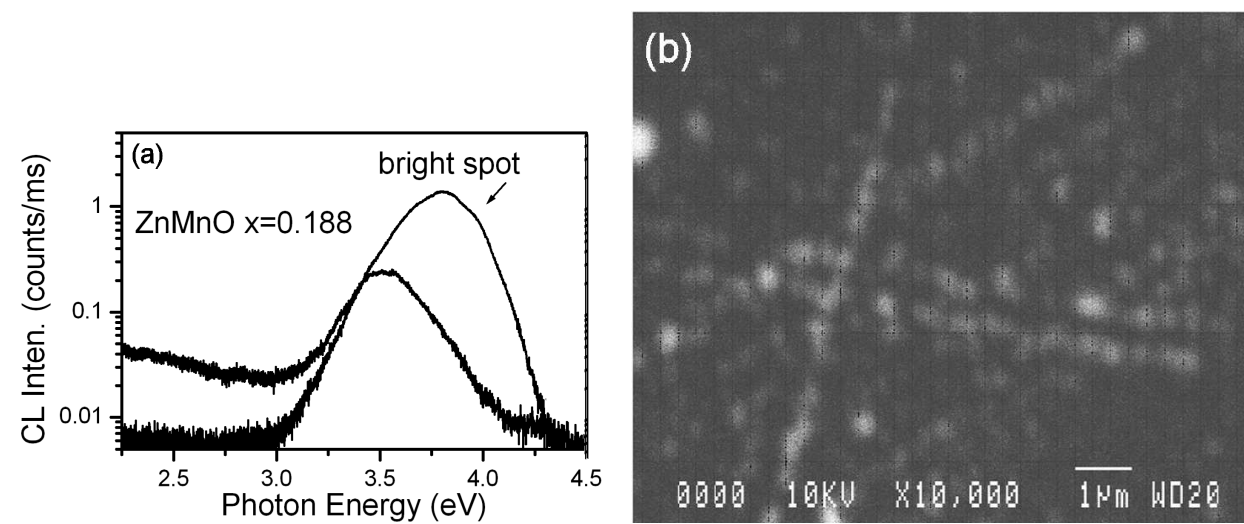

Fig. 2. CL spectrum (a) and in-plane variation of CL intensity (b) measured for ZnMnO film grown by MOVPE with $18.8 \% \mathrm{Mn}$ fraction. The spectrum and image were taken at $10 \mathrm{kV}$ and room temperature.

The $\mathrm{Mn}^{2+}$ intra-shell PL was not observed for all of the studied samples. We could also reject idea that this emission contributes to a broad red PL of $\mathrm{ZnMnO}$ (as proposed in [11]). First of all, the red PL was not observed by us for some of our ZnMnO films. Moreover, we have not observed a characteristic PL excitation (PLE) spectrum (consisting of "sharp" $\mathrm{Mn}^{2+}$ intra-shell transitions) to support such identification of the red PL. A structured PLE spectrum was observed by us e.g. in $\mathrm{ZnMnS}$ [12]. 
Magnetic properties of LT ZnMnO films were studied with SQUID and electron spin resonance. Samples grown at LT with homogeneous Mn distribution (as observed with secondary ion mass spectroscopy (SIMS) and energy distribution of X-ray (EDX)) showed paramagnetic response with only traces of antiferromagnetic coupling of close Mn-Mn pairs. These investigations will not be analyzed here. We concentrate on the results of magneto-PL investigations shown in Figs. 3 and 4. The results obtained for the MOVPE $\mathrm{ZnMnO}$ sample with $0.6 \% \mathrm{Mn}$ fraction are presented. For the DMS sample we expected very enhanced magnetic interactions. However, no magnetic field induced shift and splitting of excitonic transitions are observed (see Fig. 3), even though we could easily observe them for CdMnTe samples with a similar Mn content [13]. More surprisingly these effects are accompanied by relatively strong PL polarization (see Fig. 4), which (for $6 \mathrm{~T}$ magnetic field) is 40 times stronger than the one observed by us for the reference $\mathrm{ZnO}$ sample. We have reported recently for $\mathrm{ZnCrSe}$ samples similar observations, i.e. strong PL polarization and lack of other magneto-optical effects, and related them to spin selective carrier trapping by photo-ionized Cr centers in $\mathrm{ZnSe}$ lattice [14]. This suggests that, similarly like in GaMnN [15], Mn can be present in $\mathrm{ZnO}$ lattice in two charge states. In fact, two $\mathrm{Mn}$ states in $\mathrm{ZnMnO}$ thin films were observed in photoemission study [16], where Mn $3 p$ states show two contributions with different binding energies separated by about $4 \mathrm{eV}$.

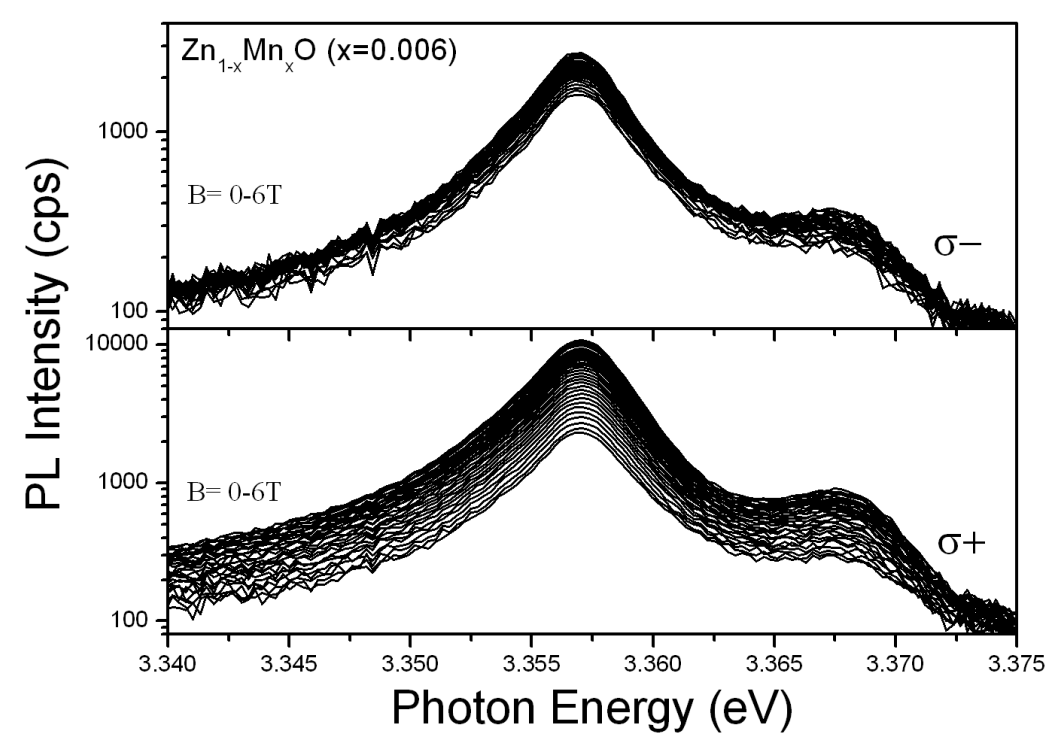

Fig. 3. Magnetic field dependence of the PL polarization measured at $2 \mathrm{~K}$ for the MOVPE sample with $0.6 \% \mathrm{Mn}$ fraction. 


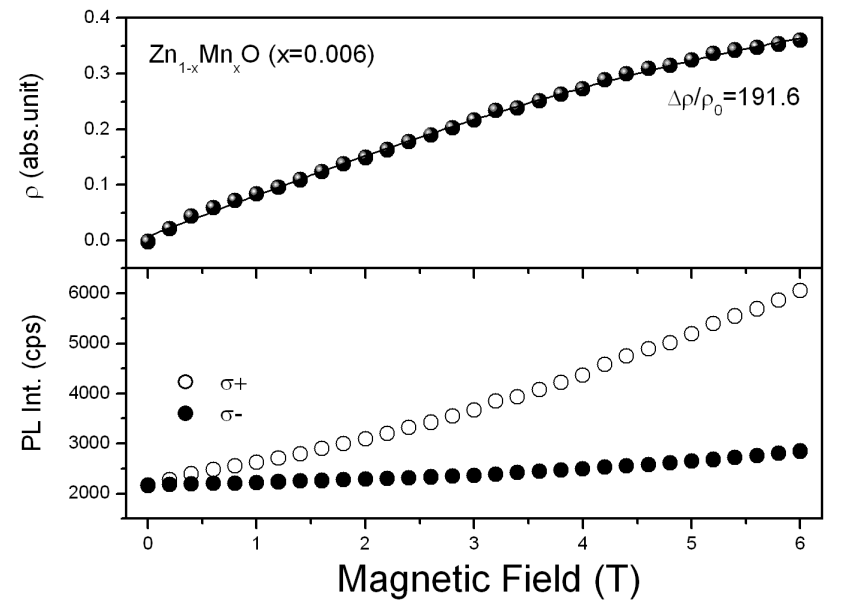

Fig. 4. Magnetic field dependence of the PL polarization rate measured at $2 \mathrm{~K}$ for the MOVPE sample with $0.6 \% \mathrm{Mn}$ fraction.

Double valence of $\mathrm{Mn}$ in $\mathrm{ZnO}$ allows to explain $\mathrm{PL} / \mathrm{CL}$ quenching in this material. "Killer action" of $\mathrm{Mn}$ in $\mathrm{ZnO}$ can be of the similar origin to the one reported by us for Fe ions on $\mathrm{ZnS}$ and $\mathrm{ZnSe}$ (see [17] and references therein). $\mathrm{PL} / \mathrm{CL}$ can be deactivated either due to efficient carrier recombination via Mn-related level (the so-called bypassing effect [17]) or to efficient Auger-type energy transfer from excitons and DAPs to Mn ions followed by Mn ionization.

Further results consistent with the above proposed mechanism of PL/CL quenching will be given in the forthcoming article [18].

\section{Conclusions}

In conclusion, we show that Mn deactivates PL/CL emission of ZnMnO. Strong PL polarization is observed even though other magneto-PL effects expected for DMS samples (shift of the excitonic lines and their splitting) are not observed. We tentatively relate this to double valence of $\mathrm{Mn}$ ions in $\mathrm{ZnO}$ lattice.

\section{Acknowledgments}

This research was partially supported by the Network "New materials and sensors for optoelectronics, information technology, energetics and medicine". M. Godlewski thanks CNRS for financial support during his stay in Versailles, France. The F-120 ALE reactor of Microchemistry was bought using SEZAM grant of Foundation for Polish Science.

\section{References}

[1] T. Dietl, H. Ohno, F. Matsukura, J. Cibert, D. Ferrand, Science 287, 1019 (2000).

[2] C. Liu, F. Yun, H. Morkoc, J. Mater. Sci., Mater. Electron. 16, 555 (2005). 
[3] A. Wójcik, K. Kopalko, M. Godlewski, E. Guziewicz, R. Jakieła, R. Minikayev, W. Paszkowicz, Appl. Phys. Lett. 89, 051907 (2006).

[4] A. Tomaszewska-Grzęda, A. Opalińska, E. Grzanka, W. Łojkowski, A. Gedanken, M. Godlewski, S. Yatsunenko, V. Osinniy, T. Story, Appl. Phys. Lett. 89, 242102 (2006).

[5] A. Wójcik, M. Godlewski, E. Guziewicz, R. Jakieła, M. Kiecana, M. Sawicki, M. Guziewicz, M. Putkonen, L. Niinistö, Y. Dumont, N. Keller, Appl. Phys. Lett. 90, 082502 (2007).

[6] E. Chikoidze, H.J. von Bardeleben, Y. Dumont, P. Galtier, J.L. Cantin, J. Appl. Phys. 97, 10D316 (2005); E. Chikoidze, Y. Dumont, F. Jomard, D. Ballutaud, P. Galtier, O. Gorochov, D. Ferrand, J. Appl. Phys. 97, 10D327 (2005).

[7] E. Chikoidze, Y. Dumont, F. Jomard, D. Ballutaud, P. Galtier, D. Ferrand, V. Sallet, O. Gorochov, Mater. Res. Bull. 41, 1038 (2006)

[8] A. Ben Mahmoud, H.J. von Bardeleben, J.L. Cantin, A. Mauger, E. Chikoidze, Y. Dumont, Phys. Rev. B 74, 115203 (2006).

[9] V.Yu. Ivanov, M. Godlewski, S. Yatsunenko, A. Khachapuridze, Z. Golacki, M. Sawicki, A. Omel'chuk, M. Bulany, A. Gorban', Phys. Status Solidi C 1, 250 (2004).

[10] V. Avrutin, N. Izuymskaya, Ü. Özgür, A. El-Shaer, H. Lee, W. Schoch, F. Reuss, V.G. Beschenkov, A.N. Pustovit, A. Che Mofor, A. Bakin, H. Morkoc, A. Waag, Superlattices Microstructures 39, 291 (2006).

[11] M. Nakayama, H. Tanaka, K. Matsuko, T. Fukushima, A. Ashida, N. Fijimura, Appl. Phys. Lett. 88, 241908 (2006).

[12] S. Yatsunenko, M. Godlewski, E. Guziewicz, M. Zalewska, A. Kłonkowski, B. Kukliński, M. Grinberg, P.J. Klar, W. Heimbrodt, to be published in J. Alloys Compd.

[13] M. Godlewski, V.Yu. Ivanov, A. Khachapuridze, S. Yatsunenko, Phys. Status Solidi B 229, 533 (2002).

[14] V.Yu. Ivanov, G. Karczewski, M. Sawicki, M. Godlewski, A.R. Omel'chuk, N.V. Zhavoronkov, A.A. Davydov, Phys. Status Solidi C 1, 961 (2004).

[15] A. Wolos, M. Palczewska, M. Zajac, J. Gosk, M. Kaminska, A. Twardowski, M. Bockowski, I. Grzegory, S. Porowski, Phys. Rev. B 69, 115210 (2004).

[16] E. Guziewicz, K. Kopalko, J. Sadowski, M. Guziewicz, Z. Gołacki, Acta Phys. Pol. A 108, 689 (2005).

[17] M. Godlewski, A. Zakrzewski, in: II-VI Semiconductor Compounds, Ed. M. Jain, World Sci., Singapore 1993, p. 205.

[18] M. Godlewski, A. Zakrzewski, A. Wójcik, K. Kopalko, S. Yatsunenko, V.Yu. Ivanov, E. Guziewicz, Y. Dumont, E. Chikoidze, to be published. 